\title{
Scientific Community and Remaining Errors, Physics Examples
}

\author{
Claude Daviau' ${ }^{1}$ Jacques Bertrand ${ }^{2}$ \\ ${ }^{1}$ Le Moulin de la Lande 44522 Pouillé-les-coteaux, France \\ ${ }^{2} 15$ Avenue Danielle Casanova, 95210 Saint Gratien, France \\ Email: daviau.claude@orange.fr, bertrandjacques-m@orange.fr
}

How to cite this paper: Daviau, C. and Bertrand, J. (2018) Scientific Community and Remaining Errors, Physics Examples. Journal of Modern Physics, 9, 250-258. https://doi.org/10.4236/jmp.2018.92017

Received: December 18, 2017

Accepted: January 21, 2018

Published: January 24, 2018

Copyright (c) 2018 by authors and Scientific Research Publishing Inc. This work is licensed under the Creative Commons Attribution International License (CC BY 4.0).

http://creativecommons.org/licenses/by/4.0/

\begin{abstract}
The scientific community controls the possible errors by a rigorous process using referees. Consequently the only possible errors are very few, they come from what anyone considers obviously true. Three of these errors are pointed here: the main one is the belief that any quantum state follows a Schrödinger equation. This induces two secondary errors: the impossibility of magnetic charges and the identification between the Lorentz group and $S L(2, \mathbb{C})$.
\end{abstract}

\section{Keywords}

Invariance Group, Schrödinger Equation, Dirac Equation, Electromagnetism, Weak Interactions, Strong Interactions, Clifford Algebra, Magnetic Monopole

\section{The Universality of the Schrödinger Equation}

Even if Erwin Schrödinger was a physicist working on General Relativity, even if Louis de Broglie introduced his wave for the movement of any particle from relativistic considerations [1], the belief that any quantum state follows a Schrödinger equation is now the basis of the axiomatic quantum theory. The first reason is the fact that the Schrödinger equation was the first one:

$$
i \hbar \frac{\partial}{\partial t} \psi=H(\psi) ; \psi=\psi(t, x, y, z)
$$

where $H$ is the Hamiltonian operator. For a single electron this operator reads:

$$
H=\frac{\hbar^{2}}{2 m_{0}}\left(\frac{\partial^{2}}{(\partial x)^{2}}+\frac{\partial^{2}}{(\partial y)^{2}}+\frac{\partial^{2}}{(\partial z)^{2}}\right)+(-e) V
$$

where $V$ is the exterior electric potential and $(-e)$ is the electric charge of the electron. Only a few months later a wave equation was obtained by Pauli 
accounting for the spin of the electron. Still a few months later Dirac gave a relativistic equation for the wave of an electron [2]:

$$
0=\left[\gamma^{\mu}\left(\partial_{\mu}+i q A_{\mu}\right)+i m\right] \psi ; \psi=\psi\left(x^{\mu}\right), x^{0}=c t ; q=\frac{e}{\hbar c} ; m=\frac{m_{0} c}{\hbar}
$$

where $A_{\mu}$ is the covariant components of the electromagnetic potential spacetime vector. This wave equation was relativistic, it was the awaited wave equation: de Broglie studied all aspects and results of this equation [3]. For high energies, the $\gamma_{\mu}$ matrices are currently chosen as following:

$$
\begin{aligned}
& \psi=\left(\begin{array}{l}
\xi \\
\eta
\end{array}\right) ; \xi=\left(\begin{array}{l}
\xi_{1} \\
\xi_{2}
\end{array}\right) ; \eta=\left(\begin{array}{l}
\eta_{1} \\
\eta_{2}
\end{array}\right), \\
& \sigma_{1}=\left(\begin{array}{ll}
0 & 1 \\
1 & 0
\end{array}\right) ; \sigma_{2}=\left(\begin{array}{cc}
0 & -i \\
i & 0
\end{array}\right) ; \sigma_{3}=\left(\begin{array}{cc}
1 & 0 \\
0 & -1
\end{array}\right), \\
& \sigma^{j}=-\hat{\sigma}^{j}=-\sigma_{j}=\hat{\sigma}_{j}, j=1,2,3 ; \\
& \gamma_{0}=\gamma^{0}=\left(\begin{array}{cc}
0 & I \\
I & 0
\end{array}\right) ; I=\sigma_{0}=\sigma^{0}=\hat{\sigma}^{0}=\hat{\sigma}_{0}=\left(\begin{array}{ll}
1 & 0 \\
0 & 1
\end{array}\right), \\
& \gamma^{j}=-\gamma_{j}=\left(\begin{array}{cc}
0 & -\sigma_{j} \\
\sigma_{j} & 0
\end{array}\right), j=1,2,3 .
\end{aligned}
$$

where $\xi_{j}=\xi_{j}(x, t), \eta_{j}=\eta_{j}(x, t)$. The $\left(\sigma_{1}, \sigma_{2}, \sigma_{3}\right)$ is a basis of our usual space, which generates the Clifford algebra $\mathrm{Cl}_{3}$ while $\left(\gamma_{0}, \gamma_{1}, \gamma_{2}, \gamma_{3}\right)$ is a basis of spacetime which generates the Clifford algebra $\mathrm{Cl}_{1,3}$. With these matrix representations the Dirac equation is equivalent to the system:

$$
\begin{aligned}
& 0=\sigma^{\mu}\left(\partial_{\mu}+i q A_{\mu}\right) \eta+i m \xi, \\
& 0=\hat{\sigma}^{\mu}\left(\partial_{\mu}+i q A_{\mu}\right) \xi+i m \eta .
\end{aligned}
$$

where $\xi$ is named the right wave and $\eta$ is named the left wave. With:

$$
\nabla=\sigma^{\mu} \partial_{\mu} ; \hat{\nabla}=\hat{\sigma}^{\mu} \partial_{\mu} ; A=\sigma^{\mu} A_{\mu} ; \hat{A}=\hat{\sigma}^{\mu} A_{\mu},
$$

and with:

$$
\begin{aligned}
& R^{1}=\sqrt{2}\left(\begin{array}{ll}
\xi & 0
\end{array}\right) ; \hat{L}^{1}=\sqrt{2}\left(\begin{array}{ll}
\eta & 0
\end{array}\right), \\
& \phi=R^{1}+L^{1}=\sqrt{2}\left(\begin{array}{cc}
\xi_{1} & -\eta_{2}^{*} \\
\xi_{2} & \eta_{1}^{*}
\end{array}\right)=\sqrt{2}\left(\begin{array}{ll}
\xi & \hat{\eta}
\end{array}\right),
\end{aligned}
$$

we get the Dirac equation in $\mathrm{Cl}_{3}$ :

$$
\nabla \hat{\phi} \sigma_{12}=q A \hat{\phi}+m \phi
$$

But this is only one of two wave equations, the wave equation of the electron and the wave equation of the positron:

$$
\begin{aligned}
& \hat{\phi}_{p}=\hat{\phi} \sigma_{1} ; \phi_{p}=-\phi \sigma_{1}, \\
& \xi_{p}=-\hat{\eta} ; \eta_{p}=\hat{\xi} \\
& \nabla \hat{\phi} \sigma_{12}=q A \hat{\phi}+m \phi, \\
& \nabla \hat{\phi}_{p} \sigma_{12}=-q A \hat{\phi}_{p}+m \phi_{p} .
\end{aligned}
$$


This is equivalent to:

$$
\begin{aligned}
& 0=\sigma^{\mu}\left(\partial_{\mu}+i q A_{\mu}\right) \eta+i m \xi, \\
& 0=\hat{\sigma}^{\mu}\left(\partial_{\mu}+i q A_{\mu}\right) \xi+i m \eta, \\
& 0=\sigma^{\mu}\left(\partial_{\mu}-i q A_{\mu}\right) \eta_{p}+i m \xi_{p}, \\
& 0=\hat{\sigma}^{\mu}\left(\partial_{\mu}-i q A_{\mu}\right) \xi_{p}+i m \eta_{p} .
\end{aligned}
$$

So the approximation coming from low velocities, where the sum $\xi+\eta$ or the difference $\xi-\eta$ is considered as negligible and is cancelled, is pure nonsense: the Schrödinger equation does not approximate the Dirac equation. It is another world, virtual, without relativistic invariance, without charge conjugation.

Therefore the postulate of axiomatic quantum physics saying that any quantum state is solution of the Schrödinger equation is false. The axiomatic form was chosen because physicists were awaiting from the mathematics the security given by the use of logics. But the real consequence of a false postulate is that anything demonstrated from the postulates may be true or may be false!

\section{The Impossibility of Magnetic Monopoles}

This impossibility is one of the false ideas coming from the previous false postulate. If any quantum state has value into the complex field and follows a Schrödinger equation, then there is only one possible phase to the wave. This phase is the electric gauge, its conservation gives the charge of the particle. There is no place for another phase.

If you disagree with this false postulate you will be able to read the Dirac wave as a function of space and time with value in the Clifford algebra of space. This algebra is non commutative, then several different phases are available. This is the starting point of the Lochak's theory of the leptonic monopole [4]-[9]. There is, aside the $i$ of the electric phase, another object with square -1 , the $\mathbf{i}=\gamma_{0} \gamma_{1} \gamma_{2} \gamma_{3}$, allowing another gauge that Lochak associates to a magnetic monopole. Actually the $\mathrm{Cl}_{3}$ algebra contains not only 2 but 4 different and independent $i$, each giving a particular gauge:

$$
i_{3}=\sigma_{12}=i \sigma_{3} ; i_{1}=\sigma_{23}=i \sigma_{1} ; i_{2}=\sigma_{31}=i \sigma_{2} ; i=\sigma_{123}=\sigma_{1} \sigma_{2} \sigma_{3} .
$$

The Lie algebra generated by these four elements is exactly the Lie algebra of the $U(1) \times S U(2)$ Lie group. This group is the gauge group of the electro-weak Weinberg-Salam model [10]. Then the chiral gauge $U(1)$ used by the Lochak's magnetic monopole is a part of the electro-weak gauge group. We have used this inclusion to study both the magnetic monopole [11] [12] [13] and the electroweak gauge. We have even extended the gauge to a geometric electro-weakchromodynamics [14]-[27].

\section{The Confusion between Different Lie Group}

The main success of the non-relativistic quantum mechanics was the explanation 
of the energy levels of the electrons in atoms. The calculation of the quantum states uses the spherical symmetry of the wave in the electric central potential. All solutions have this same symmetry, they are invariant under the $S O(3)$ group of rotations around a fixed center. The theory is well understood: if a physical system has a quantum state $\psi$ and if this system is transformed, for instance if we rotate the physical system by a rotation $R$, the quantum state becomes $\psi^{\prime}=T(\psi)$. The $T$ transformation is linear, since the wave equation is a linear one. Then the $f$ defined by:

$$
f: R \mapsto T
$$

is an homomorphism from the group $S O(3)$ of the spacial rotations (containing $R$ ) into another group $G$ (containing $T$ ) of linear transformations upon the quantum states. Physicists usually name this homomorphism a representation of $\mathrm{SO}(3)$.

The theory of homomorphisms of Lie group classifies all possible representations, using the properties of the Lie algebras of these Lie groups [28]. In the case of $S O(3)$, for each representation an integer exists. On the physical point of view this integer is the angular momentum 1 . The only important result to remember here is: this $I$ number is necessary an integer number. So the resolution of the Schrödinger equation introduced three integer numbers, $l=0,1,2,3, \cdots$, $p=0,1,2,3, \cdots$ and $m=-l,-l+1, \cdots, l-1, l$. The integer number $p$ is the degree of a radial polynomial. The principal quantum number $\mathbf{n}=l+1+p$ is then a non null integer. The energy of these states are:

$$
E=-\frac{m_{0} e^{4}}{2 \mathbf{n}^{2} \hbar^{2}} .
$$

The number of states with this energy is

$$
\sum_{l=0}^{l=\mathbf{n}-1}(2 l+1)=\mathbf{n}^{2} .
$$

The true number awaited is $2 \mathbf{n}^{2}$. Of course this was never considered as a problem, because Pauli had solved this difficulty even before the wave equation, by adding a spin number with only two possible values, $1 / 2$ and $-1 / 2$. But these non integer numbers are totally out of the frame of the Schrödinger equation, where only integer numbers are available. This should be a sufficient reason to falsify the postulate of universality of the Schrödinger equation.

Actually the problem was solved only from the Dirac equation, and in a very strange way. First the space-time was considered as included in the space algebra by letting:

$$
\begin{aligned}
& x^{0}=c t ; \vec{x}=x^{1} \sigma_{1}+x^{2} \sigma_{2}+x^{3} \sigma_{3} ; \partial_{\mu}=\frac{\partial}{\partial x^{\mu}}, \\
& x=x^{0}+\vec{x}=\left(\begin{array}{cc}
x^{0}+x^{3} & x^{1}-i x^{2} \\
x^{1}+i x^{2} & x^{0}-x^{3}
\end{array}\right) .
\end{aligned}
$$

The next oddity is: 


$$
\begin{gathered}
\hat{x}=\bar{x}=x^{0}-\vec{x} \\
\operatorname{det}(x)=x \hat{x}=x \cdot x=\left(x^{0}\right)^{2}-\vec{x}^{2}=\left(x^{0}\right)^{2}-\left(x^{1}\right)^{2}-\left(x^{2}\right)^{2}-\left(x^{3}\right)^{2}
\end{gathered}
$$

Then the square of the pseudo-norm of any space-time vector is the determinant of this vector. Let $M$ be any non null element in $\mathrm{Cl}_{3}$ and let $R$ be the transformation from space-time into itself that associates to any $x$ the spacetime element $x^{\prime}$ satisfying

$$
x^{\prime}=x^{\prime 0}+\vec{x}^{\prime}=R(x)=M x M^{\dagger} .
$$

We note, if $\operatorname{det}(M) \neq 0$ :

$$
\operatorname{det}(M)=r \mathrm{e}^{\mathrm{i} \theta}, r=|\operatorname{det}(M)| .
$$

This gives:

$$
\begin{aligned}
& \left(x^{\prime 0}\right)^{2}-\left(x^{\prime 1}\right)^{2}-\left(x^{\prime 2}\right)^{2}-\left(x^{\prime 3}\right)^{2} \\
& =\operatorname{det}\left(x^{\prime}\right)=\operatorname{det}\left(M x M^{\dagger}\right)=r \mathrm{e}^{i \theta} \operatorname{det}(x) r \mathrm{e}^{-i \theta} \\
& =r^{2}\left[\left(x^{0}\right)^{2}-\left(x^{1}\right)^{2}-\left(x^{2}\right)^{2}-\left(x^{3}\right)^{2}\right]
\end{aligned}
$$

So $R$ multiplies by $r$ any space-time distance and we name $R$ a Lorentz dilation with ratio $r$. Moreover with

$$
x^{\prime \mu}=R_{v}^{\mu} x^{v} .
$$

we get for any $M=\left(\begin{array}{ll}a & b \\ c & d\end{array}\right) \neq 0 \quad[24]$ :

$$
2 R_{0}^{0}=|a|^{2}+|b|^{2}+|c|^{2}+|d|^{2}>0
$$

$x^{\prime 0}$ has then the same sign as $x^{0}$ at the origin: $R$ conserves the arrow of time.

Moreover we get for any $\mathrm{Min}^{\mathrm{Cl}} \mathrm{l}_{3}$ (see [29] A.2.4)

$$
\operatorname{det}\left(R_{v}^{\mu}\right)=r^{4}
$$

$R$ conserves then the orientation of space-time, and since it conserves also the orientation of time it conserves also the orientation of space. The Dirac theory uses only the particular case $\operatorname{det}(M)=1$ that gives:

$$
\left(x^{\prime 0}\right)^{2}-\left(x^{\prime 1}\right)^{2}-\left(x^{\prime 2}\right)^{2}-\left(x^{\prime 3}\right)^{2}=\left[\left(x^{0}\right)^{2}-\left(x^{1}\right)^{2}-\left(x^{2}\right)^{2}-\left(x^{3}\right)^{2}\right] ; \operatorname{det}\left(R_{v}^{\mu}\right)=1 .
$$

And $R$ is a Lorentz transformation belonging to the restricted Lorentz group made of the transformations conserving the orientation of space and time. The Dirac wave satisfies:

$$
\xi^{\prime}=\xi^{\prime}\left(x^{\prime}\right)=M \xi=M \xi(x) ; \eta^{\prime}=\eta^{\prime}\left(x^{\prime}\right)=\hat{M} \eta=\hat{M} \eta(x) .
$$

And we also get, with $\bar{M}=\hat{M}^{\dagger}$ :

$$
\phi^{\prime}=M \phi ; \nabla=\bar{M} \nabla^{\prime} \hat{M} .
$$


When $\operatorname{det}(M)=1$ and from the general relation $\operatorname{det}(M)=M \bar{M}$ we get $\bar{M}=M^{-1}$ and (8) gives:

$$
\begin{aligned}
0 & =\bar{M} \nabla^{\prime} \hat{M} \hat{\phi} \sigma_{21}+q \bar{M} A^{\prime} \hat{M} \hat{\phi}+m M^{-1} M \phi \\
& =M^{-1}\left[\nabla^{\prime} \hat{\phi}^{\prime} \sigma_{21}+q A^{\prime} \hat{\phi}^{\prime}+m \phi^{\prime}\right], \\
0 & =\nabla^{\prime} \hat{\phi}^{\prime} \sigma_{21}+q A^{\prime} \hat{\phi}^{\prime}+m \varphi^{\prime} \Leftrightarrow 0=\nabla \hat{\phi} \sigma_{21}+q A \hat{\phi}+m \phi .
\end{aligned}
$$

This is why the Dirac equation is said invariant under a Lorentz transformation. Nothing is wrong there, except that the Dirac equation is form invariant under the set of the $M$, that is $S L(2, \mathbb{C})$, not the set of the $R$ which is the subgroup $\hat{\mathcal{L}_{+}}$of the Lorentz group. In the case of the initial formulation of the Dirac theory, it is necessary to use instead of $M$ the $N$ verifying

$$
N=\left(\begin{array}{cc}
M & 0 \\
0 & \hat{M}
\end{array}\right) .
$$

Since the conjugation $M \mapsto \hat{M}$ is the main automorphism of $\mathrm{Cl}_{3}$ the group of the $N$ is isomorphic to the group of the $M$.

Next for any $M$ satisfying (20), (21), (23) and (25) we have for each value $v=0,1,2,3$ (a proof is in [30] A.2.2):

$$
R_{\mu}^{v} \gamma^{\mu}=\tilde{N} \gamma^{v} N
$$

We also have

$$
\partial_{v}^{\prime}=\frac{\partial}{\partial x^{\prime \nu}} ; \partial_{\mu}=R_{\mu}^{v} \partial_{v}^{\prime} ; A_{\mu}=R_{\mu}^{v} A^{\prime},
$$

and so we get:

$$
\begin{aligned}
0 & =\left[\gamma^{\mu}\left(\partial_{\mu}+i q A_{\mu}\right)+i m\right] \psi \\
& =\left[\gamma^{\mu} R_{\mu}^{v}\left(\partial_{v}^{\prime}+i q A_{\nu}^{\prime}\right)+i m\right] \psi \\
& =\left[\tilde{N} \gamma^{v} N\left(\partial_{v}^{\prime}+i q A_{\nu}^{\prime}\right)+i m\right] \psi .
\end{aligned}
$$

If we restrict $M$ to $S L(2, \mathbb{C})$, we get $M \bar{M}=\operatorname{det}(M)=1$, so $\bar{M}=M^{-1}$ and $\tilde{N}=N^{-1}$ which allows us to write

$$
\left[\tilde{N} \gamma^{v} N\left(\partial_{v}^{\prime}+i q A_{v}^{\prime}\right)+i m\right] \psi=N^{-1}\left[\gamma^{v}\left(\partial_{v}^{\prime}+i q A^{\prime}\right)+i m\right] N \psi .
$$

So the Dirac theory supposes:

$$
\psi^{\prime}=N \psi,
$$

we can easily see that it is equivalent to (25) and we get

$$
0=\left[\gamma^{\mu}\left(\partial_{\mu}+i q A_{\mu}\right)+i m\right] \psi=N^{-1}\left[\gamma^{\mu}\left(\partial_{\mu}^{\prime}+i q A_{\mu}^{\prime}\right)+i m\right] \psi^{\prime} .
$$

Then we must say that, even in the first form of the Dirac theory, the Dirac wave is invariant not under the Lorentz transformations, but under the $S L(2, \mathbb{C})$ Lie group. This is more easy to see if we extend the invariance to the $G L(2, \mathbb{C})=\mathrm{Cl}_{3}^{*}$ group made of any invertible $\mathrm{M}$ in $\mathrm{Cl}_{3}$. This invariance is possible because (20), (23) and (26) are general and do not need the restrictive condition $\operatorname{det}(M)=1$. This allows not only an extended invariance, but also an 
extended Lagrangian density and an extended wave equation accounting for all elements of one generation [24].

The resolution of the Dirac equation in the case of the $\mathrm{H}$ atom is possible [31], like with the Schrödinger equation, by separating the variables in spherical coordinates. Simply now it is much more difficult because we have a system of angular equations and a system of radial equations. The first step of the separation introduces a constant of separation for the time that is the energy of the stationary state and another constant $\lambda$ that is the magnetic number. Next the separation between the angular and the radial system introduces a numeric constant $\kappa$. To get well defined functions $\kappa$ cannot be zero and to get a density of probability $\kappa$ must be an integer. Then $\kappa= \pm 1, \pm 2, \pm 3, \cdots$. The solution is automatically a proper vector of the $J_{3}$ momentum operator with the proper value $\lambda$ and a proper vector of the $J^{2}$ operator with the proper value $j(j+1)$ where $j=|\kappa|-\frac{1}{2}$. And the angular functions are well defined only if $\lambda=-j,-j+1, \cdots, j-1, j$. A last integer $n=0,1,2, \cdots$ appears necessary, as degree of radial polynomial functions, so as to get a density of probability. The energy levels are:

$$
E=\frac{m_{0} c^{2}}{\sqrt{1+\alpha^{2} /(s+n)^{2}}} ; s=\sqrt{\kappa^{2}-\alpha^{2}},
$$

where $\alpha$ is the fine structure constant. Next there are none state to count with $\kappa<0$ and $n=0$, this gives for $\kappa>0$ a number of states equal to $\mathbf{n}(\mathbf{n}+1)$ and for $\kappa<0$ a number of states equal to $\mathbf{n}(\mathbf{n}-1)$ where $\mathbf{n}=|\kappa|+n$. Finally the total number of states with principal quantum number $\mathbf{n}$ is $\mathbf{n}(\mathbf{n}+1)+\mathbf{n}(\mathbf{n}-1)=2 \mathbf{n}^{2}$. The result was correctly guessed from the reasoning of Pauli. Today, compared with the true complicated explanation, this presentation by spin up and down is only a tale for children!

\section{Concluding Remarks}

The link between the main error in section 1 and the last one is the abuse of methods of approximations in quantum mechanics. The first study of the relativistic invariance of the Dirac equation used infinitesimal operators next the exponentiation of these operators. On the contrary we used here exact and general calculations completely without the $\approx$ symbol.

The false postulate of the universality of the Schrödinger wave also induced the idea that any true physical formalism was necessary based on the complex field. If you look at the Dirac equation all seem actually based on the complex field. But this is mainly an accident, a coincidence between the Clifford algebra $\mathrm{Cl}_{3}$ of the real space with dimension $2^{3}$ on $\mathbb{R}$ and the complex space of the $2 \times 2$ matrices with dimension 4 on the $\mathbb{C}$ field, which is then also an algebra on the real field with dimension 8 . This isomorphism is not an isomorphism of complex algebras, only of real algebras. The generator of the electric gauge for the Dirac Equation (8) is $i_{3}=i \sigma_{3}$. The fact that several different $i$ exist in 
quantum physics is totally incredible for any believer of the axiomatic quantum theory [32].

\section{Acknowledgements}

Sincere thanks to the members of JMP for their professional performance, and for the use of Tex.

\section{References}

[1] de Broglie, L. (1924) Annales de la Fondation Louis de Broglie, 17, 1-108.

[2] Drac, P.A.M. (1928) Proceedings of the Royal Society London, 117, 610-624. https://doi.org/10.1098/rspa.1928.0023

[3] de Broglie, L. (1934) L'Electron magntique. Herman, Singapore.

[4] Lochak, G. (1983) Annales de la Fondation Louis de Broglie, 9, 4.

[5] Lochak, G. (1983) Annales de la Fondation Louis de Broglie, 10, 1.

[6] Lochak, G. (1985) International Journal of Theoretical Physics, 24, 1019-1050. https://doi.org/10.1007/BF00670815

[7] Lochak, G. (2001) Annales de la Fondation Louis de Broglie, 26, 31-42.

[8] Lochak, G. (1986) Annales de la Fondation Louis de Broglie, 29, 297-316.

[9] Lochak, G. (2006) Annales de la Fondation Louis de Broglie, 31, 193-206.

[10] Weinberg, S. (1967) Physical Review Letters, 19, 1264-1266. https://doi.org/10.1103/PhysRevLett.19.1264

[11] Priem, D., Daviau, C. and Racineux, G. (2009) Annales de la Fondation Louis de Broglie, 34, 103.

[12] Daviau, C., Fargue, D., Priem, D. and Racineux. G. (2013) Annales de la Fondation Louis de Broglie, 38, 139-153.

[13] Daviau, C., Priem, D. and Racineux. G. (2013) Annales de la Fondation Louis de Broglie, 38, 189-194.

[14] Daviau, C. (2013) Advances in Imaging and Electron Physic, 179, 1-137. https://doi.org/10.1016/B978-0-12-407700-3.00001-6

[15] Daviau, C. (2015) Annales de la Fondation Louis de Broglie, 40, 113-138.

[16] Daviau, C. and Bertrand, J. (2014) Journal of Modern Physics, 5, 1001-1022. https://doi.org/10.4236/jmp.2014.511102

[17] Daviau, C. and Bertrand, J. (2014) Journal of Modern Physics, 5, 2149-2173. https://doi.org/10.4236/jmp.2014.518210

[18] Daviau, C. and Bertrand, J. (2014) New Insights in the Standard Model of Quantum Physics in Clifford Algebra, JePublie, Pouillé-les-coteaux. http://hal.archives-ouvertes.fr/hal-00907848

[19] Daviau, C. and Bertrand, J. (2015) Annales de la Fondation Louis de Broglie, 40, 181-209.

[20] Daviau, C. and Bertrand, J. (2015) Journal of Modern Physics, 6, 2080-2092. https://doi.org/10.4236/jmp.2015.614215

[21] Daviau, C. and Bertrand, J. (2015) Journal of Applied Mathematics and Physics, 3, 46-61.

[22] Daviau, C. and Bertrand, J. (2015) Journal of Modern Physics, 6, 1647-1656. https://doi.org/10.4236/jmp.2015.611166 
[23] Daviau, C. and Bertrand, J. (2016) Annales de la Fondation Louis de Broglie, 41, 73-97.

[24] Daviau, C. and Bertrand, J. (2016) The Standard Model of Quantum Physics in Clifford Algebra. World Scientific, Singapore.

[25] Daviau, C., Bertrand, J. and Girardot, D. (2016) Journal of Modern Physics, 7, 1568-1590. https://doi.org/10.4236/jmp.2016.712143

[26] Daviau, C., Bertrand, J. and Girardot, D. (2016) Journal of Modern Physics, 6, 2398-2417. https://doi.org/10.4236/jmp.2016.716207

[27] Daviau, C., Bertrand, J., Girardot, D. and Socroun, T. (2017) Annales de la Fondation Louis de Broglie, 42, 2.

[28] Bacry, H.P. (19671) Leçons sur la Théorie des Groupes et les Symétries des Particules Elémentaire, Gordon and Breach, Paris Londres New York.

[29] Daviau, C. (2012) Double Space-Time and more, JePublie, Pouillé-les-coteaux.

[30] Daviau, C. (2011) L'espace-temps double, JePublie, Pouillé-les-coteaux.

[31] Daviau, C. (1997) Advances in Applied Clifford Algebras, 7, 175-194.

[32] Daviau, C. (2017) Dialogue pour une nouvelle physique, Ed. St Honoré, Paris. 